\title{
3D Parallel Monte Carlo Simulation of GaAs MESFETs
}

\author{
S. PENNATHUR, CAN K. SANDALCI, ÇETIN K. KOÇ and S.M. GOODNICK \\ Department of Electrical and Computer Engineering, Oregon State University, Corvallis, OR 97331, USA
}

\begin{abstract}
We have investigated three-dimensional (3D) effects in sub-micron GaAs MESFETs using a parallel Monte Carlo device simulator, PMC-3D [1]. The parallel algorithm couples a standard Monte Carlo particle simulator for the Boltzmann equation with a 3D Poisson solver using spatial decomposition of the device domain onto separate processors. The scaling properties of the small signal parameters have been simulated for both the gate width in the third dimension as well as the gate length. For realistic 3D device structures, we find that the main performance bottleneck is the Poisson solver rather than the Monte Carlo particle simulator for the parallel successive overrelaxation (SOR) scheme employed in [1]. A parallel multigrid algorithm is reported and compared to the previous SOR implementation, where considerable speedup is obtained.
\end{abstract}

Keywords: Device Simulation, Monte Carlo, Multiprocessor, Transport, Parallel Computing.

\section{INTRODUCTION}

As semiconductor device dimensions continue to shrink in ultra-large scale integration technology, there is an increasing need for full, three-dimensional (3D) device models to accurately represent the physical characteristics of the device. Solution of the Boltzmann equation using Monte Carlo methods is currently one of the most widespread techniques used in device simulation at this level of modeling [2]. In the Monte Carlo method, the motion of charge carriers (electrons and holes) is assumed to be given by classical trajectories interrupted by random, instantaneous, scattering events which change the energy and momentum of the particles. In a device simulation, the forces determining the free trajectories are determined by the electric fields obtained by solving Poisson's equation on a mesh over the device domain. The random scattering events are generated stochastically using a random number generator and the quantum mechanical scattering probabilities for all possible mechanisms in the semiconductor. In a Monte Carlo device simulation, the solution of the particle motion is synchronized with the solution of Poisson's equation so as to provide an accurate representation of the time dependent evolution of the fields in the semiconductor.

Parallel or multiprocessor computers provide some relief to the computational requirements of Monte Carlo device simulation. We have previously developed a parallel 3D Monte Carlo device simulator, PMC-3D [1], which was implemented on the distributed-memory nCUBE multiprocessor. In this algorithm, a subspace decomposition of the 3D device domain was performed, in which the particles and mesh nodes were distributed in a load-balanced way among the individual processors. During each time step, the particle motion and field calculation is performed locally, and the results communicated to neighboring processors at the end of the time step. In 

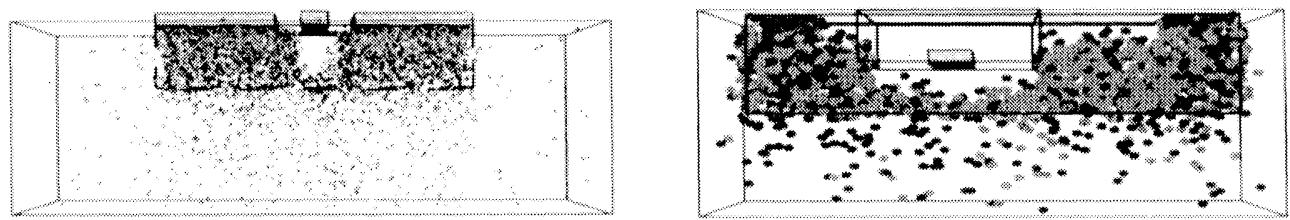

FIGURE 1 Particle distribution in steady-state for a planar GaAs MESFET device $\left(\mathrm{L}_{g}=0.25 \mu \mathrm{m}\right)$ and a recessed-gate device $\left(\mathrm{L}_{g}=0.5 \mu \mathrm{m}\right)$

order to parallelize the solution of Poisson's equation in this initial implementation, an iterative successive over relaxation (SOR) method with a red-black ordering scheme was used. We have obtained good efficiencies using this algorithm, up to $70 \%$ with 512 processors.

\section{D MESFET SIMULATION}

As an application of PMC-3D, we have studied the scaling behavior of GaAs MESFETs as both the length and the width of the gate are scaled. Figure 1 shows the result for the particle distribution for a planar and a recessed gate structure under bias. The Monte Carlo model for bulk GaAs includes all the pertinent scattering mechanisms and a three-valley conduction band. Dirichlet boundary conditions are assumed for the three electrodes, and Neumann boundary conditions are assumed elsewhere, except on the top surface wherein Fermi-level pinning of the GaAs-air interface is assumed. The MESFET structure shown in Fig. 1 is doped $2 \times 10^{17} \mathrm{~cm}^{-3}$ in the region shown, and is surrounded by a semi-insulating substrate which here is assumed doped p-type at $1 \times$ $10^{15} \mathrm{~cm}^{-3}$.

We have studied small signal parameters such as the transconductance and voltage gain as a function of gate width and length scaling. Results for the transconductance are shown in Fig. 2. As can be seen, significant deviations from the linear scaling relation with gate length are observed as the gate width is reduced. The major effect responsible for this deviation is a stronger shift in the threshold voltage with gate length for narrow gate widths which moves the operating characteristics off of the optimum operating point.

\section{THE MULTIGRID METHOD}

We have found in the above simulations that the parallel SOR solver consumes over $90 \%$ of the computation time in a typical run, motivating the search for a more efficient solution method for Poisson's equation. The multigrid technique is a well-established approach for solving ordinary and partial differential equations. Its main advantage over other iterative methods like the SOR is that it is immune to increasing grid point numbers and/or more accurate convergence thresholds [3]. In the multigrid method, the convergence of the Gauss-Seidel iteration is accelerated through the use of coarser grids on which the residual is solved. Improvement of the $2 \mathrm{D}$ Poisson-Monte Carlo algorithm has been reported by Saraniti et al. using multigrid methods [4]. in which

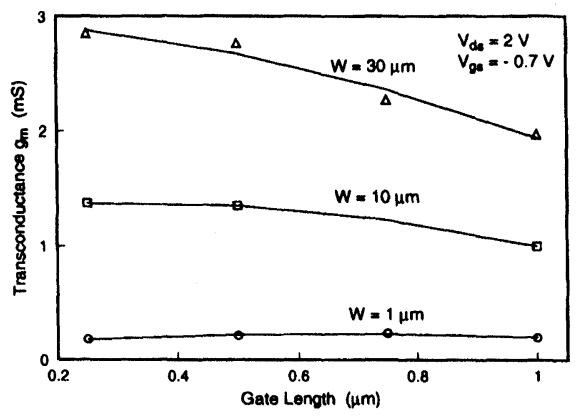

FIGURE 2 DC Transconductance variation with gate length for three different gate widths for the planar device shown in Fig. 1 
speedups of 10-20 times were reported for a sequential code compared to the SOR method.

The first task in the multigrid method is to create a hierarchical set of grids ranging from the densest $\Omega_{n}$ to the coarsest possible $\Omega_{k}$. The coarsening factor we used is $1 / 2$, which implies that the grid spacing of $\Omega_{n-1}$ is twice as big as the grid spacing of $\Omega_{n}$. Fig. 3 illustrates the two dimensional representation of the multiprocessor coarsening scheme. Choosing the number of points of the form $2^{k}+1$ for all three directions (but not necessarily with equal $k$ values) improves the convergence ratio of the Poisson Solver.

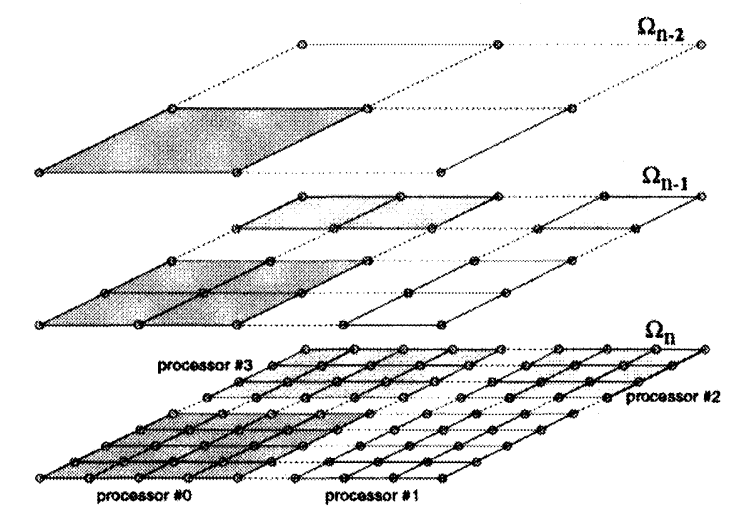

FIGURE 3 Two dimensional representation of the multiprocessor coarsening scheme. Here $\Omega_{n}$ is the densest, $\Omega_{n-1}$ is the next coarser, and $\Omega_{n-2}$ is the coarsest grid

The main goal of the relaxation scheme is to reduce the high frequency components of the error on any given grid. There can be several suitable relaxation schemes for a specific problem depending on the boundary conditions and/or coarsening method. In this implementation, we chose to use a pointwise Gauss-Seidel relaxation scheme. As the multigrid solver is designed to be a replacement for the former SOR solver, we chose to use a pointwise red-black ordered Gauss-Seidel relaxation scheme and restricted the grids to be homogenous and uniformly spaced along all three dimensions.

Several parallel implementations of the multigrid method has been reported in the literature $[5,6]$. Our parallelization of the multigrid code is essentially the same as the former SOR implementation. The partitioning and the communication routines are extended to service the hierarchical grids, hence the communi- cation pattern is preserved. Since the Gauss-Seidel relaxation operator is simply the SOR with $\omega=1$, the communication pattern of the smoothing operator remains unchanged [1].

To test the efficiency of the parallel multigrid solver, we have performed timing experiments on the planar MESFET geometry shown in Fig. 1, and compared to the previous SOR implementation. The comparisons use a fixed number of 20,000 particles for the Monte Carlo phase for a grid size of $129 \times 65 \times 33$. For these particular simulation conditions, one can see from Table I that the Poisson solver is already using $87 \%$ of the time in the simulation for the lowest accuracy, and even larger fractions as the accuracy increases. As the accuracy of the Poisson solver is increased, the speedup of the multigrid solver compared to the SOR solver increases. This effect is due to the increased efficiency of the multigrid solver in reducing the number of iterations necessary to reach convergence, which increase as the accuracy increases. Typically an accuracy of $10^{-6}$ is used in our simulations which translates to a speedup of 7.3 times for the Poisson phase and 6 times for the entire simulation which is a considerable time saving. For the same code run sequentially on an HP workstation for an accuracy of $10^{-6}$, the speedup of the multigrid 13 times faster than the SOR. The reason for the reduction in efficiency of the parallel multigrid compared to the sequential version is the additional communication overhead that is incurred with the additional grids. We are currently investigating methods of reducing this overhead and thus achieve the full advantage of using multigrids in PMC-3D.

TABLE I The timings of the PMC-3D device simulator with SOR and MG solvers. The simulation is run for 100 time steps for 20,000 particles with different convergence thresholds on a $129 \times 65 \times 33$ homogenous grid with uniform grid spacings on a 32 node nCUBE multiprocessor. The timings are in seconds

\begin{tabular}{ccccccc}
\hline \multirow{2}{*}{ Threshold } & \multicolumn{2}{c}{ PMC-3D with SOR } & \multicolumn{2}{c}{ PMC-3D with MG } & \multicolumn{2}{c}{ Speedup } \\
\cline { 2 - 7 } & Poisson & Total & Poisson & Total & \multicolumn{3}{c}{ Poisson } & Total \\
\cline { 2 - 7 } $10^{-3}$ & 2917.611 & 3340.020 & 596.367 & 1121.035 & 4.9 & 3.0 \\
$10^{-6}$ & 15093.156 & 15515.990 & 2064.199 & 2589.482 & 7.3 & 6.0 \\
$10^{-9}$ & 31167.143 & 31653.002 & 3486.319 & 4011.031 & 8.94 & 7.89 \\
\hline
\end{tabular}




\section{Acknowledgements}

The authors would like to thank M. Saraniti for helpful discussions in relation to this work. This research is supported in part by the National Science Foundation under grant ECS-9312240. One of us (SMG) further acknowledges the support of the Alexander von Humboldt foundation for support.

\section{References}

[1] U. A. Ranawake, C. Huster, P. M. Lenders, and S. M. Goodnick, "PMC-3D: A parallel three-dimensional Monte Carlo semiconductor device simulator," IEEE Transactions on Computer-Aided Design of Integrated Circuits and Systems, 13(6): 712-724, 1994.

[2] C. Jacoboni and P. Lugli, The Monte Carlo Method for Semiconductor Device Simulation. Vienna, Austria: Springer-Verlag, 1989.

[3] W. Hackbusch, Multi-Grid Methods and Applications, Berlin: Springer-Verlag, 1985.

[4] M. Saraniti, A. Rein, G. Zandler, P. Vogl and P. Lugli, "An efficient multigrid Poisson solver for device simulations," to be published in IEEE Transactions on Computer-Aided Design of Integrated Circuits and Systems, 1996.

[5] A. Brandt, "Multigrid solvers on parallel computers," In M.H. Schultz, editor, Elliptic Problem Solvers, pages 39-84 New York, Academic Press, 1981.

[6] A. Greenbaum, "A multigrid method for multiprocessors," Applied Mathematics and Computation, 19:75-88, 1986.

\section{Biographies}

Shankar Pennathur received his Ph.D in electrical engineering from Oregon State University in 1995. Since 1995, he has worked at Cirrus Logic on semiconductor device and circuit modeling. (Current address : Cirrus Logic, 3100, West Warren Avenue, Fremont, CA 94538. Phone: (510) 623-8300 Email: shankarp@corp.cirrus.com)

Stephen M. Goodnick has served on the faculty of the Department of Electrical and Computer Engineering at Oregon State University since 1986 where is currently a Professor. His main research interests involve transport in semiconductor devices. (Principal author, FAX 541-737-1300 Phone : 541-737-2970 email: goodnick@ece.orst.edu)

Can K. Sandalci has received his B.S. (1994) degree in computer engineering from Istanbul Technical University, Istanbul Turkey. Since 1995, he has been enrolled in the Ph.D. program at Oregon State University, Corvallis, OR.

Çetin K. Koç has served on the faculty of the Department of Electrical and Computer Engineering at Oregon State University since 1992 where is currently an Associate Professor. His main research interests involve parallel computation, computer arithmetic, computer algebra, and cryptography. 

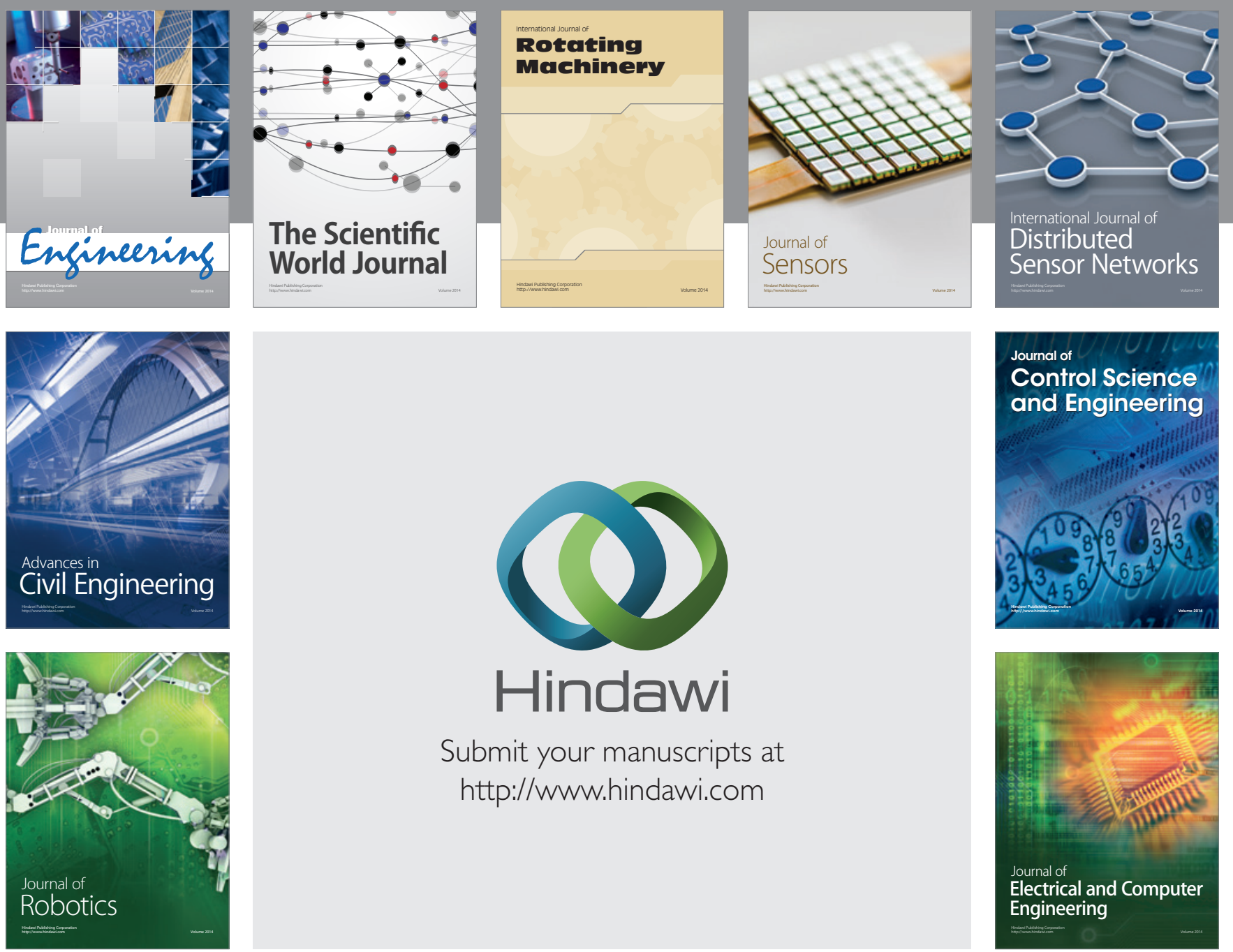

Submit your manuscripts at

http://www.hindawi.com
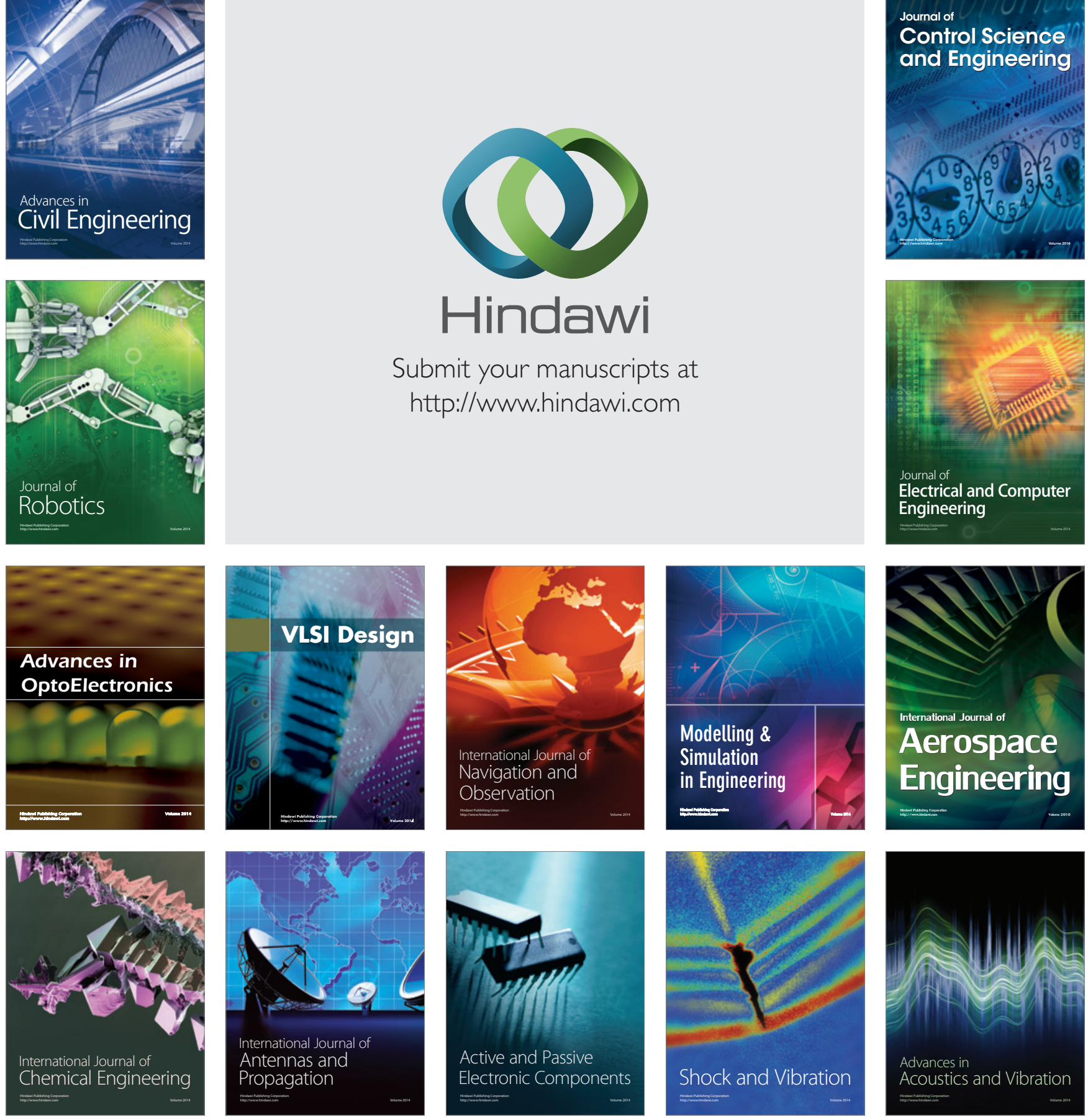